\title{
(EERSM): Energy-Efficient Multi-Hop Routing Technique in Wireless Sensor Networks Based on Combination between Stationary and Mobile Nodes
}

\author{
Fawaz Alassery \\ Computer Engineering Department, Taif University, Taif, Saudi Arabia \\ Email: falasser@tu.edu.sa
}

How to cite this paper: Alassery, F. (2019) (EERSM): Energy-Efficient Multi-Hop Routing Technique in Wireless Sensor Networks Based on Combination between Stationary and Mobile Nodes. Journal of Computer and Communications, 7, 31-52.

https://doi.org/10.4236/jcc.2019.74004

Received: March 3, 2019

Accepted: April 16, 2019

Published: April 19, 2019

Copyright $\odot 2019$ by author(s) and Scientific Research Publishing Inc. This work is licensed under the Creative Commons Attribution International License (CC BY 4.0).

http://creativecommons.org/licenses/by/4.0/

\begin{abstract}
In Wireless Sensor Network (WSNs), sensor nodes collect data and send them to a Base Station (BS) for further processing. One of the most issues in WSNs that researchers have proposed a hundred of technique to solve its impact is the energy constraint since sensor nodes have small battery, small memory and less data processing with low computational capabilities. However, many researches efforts have focused on how to prolong the battery lifetime of sensor nodes by proposing different routing, MAC, localization, data aggregation, topology construction techniques. In this paper, we will focus on routing techniques which aim to prolonging the network lifetime. Hence, we propose an Energy-Efficient Routing technique in WSNs based on Stationary and Mobile nodes (EERSM). Sensing filed is divided into intersected circles which contain Mobile Nodes (MN). The proposed data aggregation technique via the circular topology will eliminate the redundant data to be sent to the Base Station (BS). MN in each circle will rout packets for their source nodes, and move to the intersected area where another $\mathrm{MN}$ is waiting (sleep mode) to receive the transmitted packet, and then the packet will be delivered to the next intersected area until the packet is arrived to the BS. Our proposed EERSM technique is simulated using MATLAB and compared with conventional multi-hop techniques under different network models and scenarios. In the simulation, we will show how the proposed EERSM technique overcomes many routing protocols in terms of the number of hops counted when sending packets from a source node to the destination (i.e. BS), the average residual energy, number of sent packets to the BS, and the number of a live sensor nodes verse the simulation rounds.
\end{abstract}




\section{Keywords}

Energy Efficiency in WSNs, Data Aggregation of WSNs, Mobile Nodes in WSNs, Multi-Hop Routing in WSNs, Power Efficient Techniques

\section{Introduction}

Wireless Sensor Networks (WSNs) composed a hundred of sensor nodes which are deployed in a harsh environment [1]. Sensor nodes have many capabilities such as sensing, gathering, data processing and storing. These capabilities depend on batteries of sensor nodes which need to be extended in order to satisfy WSNs applications [1]. Nodes sense or monitor the sensing filed, gather data and process them before sending packets to the BS. All of these mechanisms consume power which contradicts with main feature of WSNs which have limited power resources and lossy networks [1]. Usually, wireless sensor nodes communicate with each other using multi-hop transmission over radio communication channels in order to send their packets from a source node to a destination [2]. Thus, the sensor nodes contain transceiver module, processing module and power module [2].

Reliability, real-time performance of WSNs, safety/accuracy of transmitted data and the network lifetime are the major challenges in WSNs [2]. The reliability in WSNs is the measure of the number of correct packets which are arrived correctly to the destination (i.e. the BS). Authors in [3] analyzed the reliability of WSNs over different topologies such as clustering the sensor nodes into groups. They also studied the strategies that affect reliable packets transmission such as multiple sink nodes in the sensing filed and single/multipath transmission.

For the real-time performance in WSNs, authors in [4] proposed a framework which enhances the Quality-of-Experience (QoE) for Wireless Multimedia Sensor Networks (WMSNs). The main objective of the proposed framework is to increase the network throughput and avoid dropping packets when transferred to a destination. For the safety and accuracy of data in WSNs, many applications such as in military require data to be accurate in order to avoid unexpected circumstances and damages in a battle filed as an example. Two machine learning approaches for fault detection of transmitted packets are proposed in [5] for the purpose of increasing accuracy of energy management applications. For the network lifetime in WSNs, it is known that WSNs have limited power resources with low processing capabilities, and the battery lifetime of a sensor node should be extended [1]. Figure 1 summarizes some WSNs challenges.

WSNs have been utilized in many applications such as Military missions, Agricultural missions, Industrial WSNs, smart houses, home appliances, healthcare monitoring, disaster monitoring, traffic and transportation monitoring and the environmental sensing [2].

For WSNs in military applications, authors in [6] proposed a secure 
Reliability
(Correct packets arrival)

Real-Time Performance Safety/Accuracy of packets (High throughput, QoS, QoE) (Fault detection of errors)

Network lifetime

(Power efficient techniques: routing, MAC protocols, data aggregations)

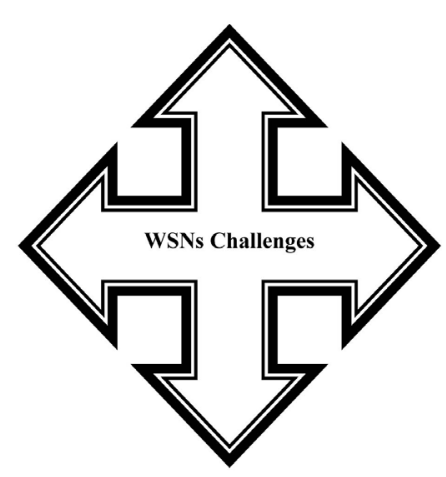

Figure 1. Some WSNs challenges.

framework for military applications based on WSNs. This framework has different modules such as cryptographic algorithm, operation modes and MAC protocols. The advantages of the proposed algorithm are to increase messages integration and data confidentiality. Authors in [7] proposed WSNs architecture for tactical requirements of a remote and large scale sensing field for military application. They divided the sensing filed into clusters and proposed a Cluster Head $(\mathrm{CH})$ algorithm for a node selection to be the master for transmission. Scalability, real-time communication and affordability of WSNs in military application are investigated in [8].

For WSNs in agricultural missions, authors in [9] proposed a low cost turbidity sensor design for the purpose of monitoring water quality online. The idea is based designing sensor nodes which utilize orthogonal scattered light and transmitted light detection principle. The proposed sensor design shows accurate readings in comparison with the commercial sensor nodes. Authors in [10] employed WSNs as data collection tool and decision system which help farmers for their manual or automated irrigation activities.

For WSNs in industrial applications, authors in [11] proposed a system model which measures the high temperature based on WSNs in an industrial environment. Semi-passive chip is designed and vacuum packaging is described for the purpose of saving electronics against high temperature which cause damages. In [12], MAC protocol for time critical industrial WSNs is proposed in order to ensure delivery of packets before the deadline bounds (i.e. deterministic channel access). The proposed MAC protocol shows better performance than TDMA based WSNs. Author in [13] proposed a routing algorithm in industrial WSNs to ensure Quality of Services (QoS) for high and low reliability packets delivery. The proposed algorithm achieves a reasonable traffic balancing which prevents network fragmentations.

For smart homes based WSNs and their related appliances, quality of life for elderly people in smart houses can be improved using a proposed system that monitors electricity and different house devices. The system allows smart and 
remote access to these devices which can be turned on using Android cell phones [14]. Authors in [15] measured the amount of energy consumption at a smart home using ZigBee WSNs. They proposed an interface which can shows the value of consumed energy either numerically or graphically for automated houses. A combination between WSNs and wireless smart phone is used to prevent accidents of disabled people at smart houses [16]. Sensor nodes are distributed in different rooms and detect potential hazards. After that, sensors send notifications to smart cell phones in order to take actions.

In terms of healthcare monitoring systems based WSNs, authors in [17] proposed a positioning system (i.e. WSN4QoL) for energy efficient monitoring of patients in indoor homes. In that system, patients can be located in indoor environment with high level of accuracy without extra hardware requirements. In Body Area Networks (BANs), using smart phones to collect packets and working as a sink node to send them to the back-end servers is proposed in [18].

For traffic monitoring based on WSNs, authors in [19] uses the graph theory for intelligent traffic monitoring in smart cities. Sensor nodes are distributed around traffic signals, reads and parking. Authors in [20] proposed data centric routing technique for packets delivery for the purpose of traffic monitoring in small scale urban areas.

The objective of this paper is to show how our proposed routing technique achieves high energy efficiency in WSNs in comparison with traditional routing techniques. The proposed routing technique has judicious selection of scenarios and parameters which make WSNs design more flexible and adaptable. In particular, the main contributions of this paper are twofold:

1) Designing a WSN based on multi-hop routing to achieve high level of scalability network.

2) Proposing a new network transmission scenario which includes fixed and mobile nodes for the purpose of fast packets delivery which can be applicable for emergency situations. The Proposing technique has a new data transmission model which ensures that sensor nodes in the same cluster don't send the same packets which leads to further power saving and extending the battery lifetime of sensor nodes.

The rest of the paper is organized as follows. Section 2 discusses some related works. Section 3 discusses the network transmission scenario of the proposed EERSM technique. Our proposed EERSM algorithm is explained deeply in Section 4. The proposed EERSM algorithm examples are discussed in Section 5. The simulation results for different performance parameters (i.e. the average residual energy, the number of sent packets to the BS, and the number of alive sensor nodes) are explained in Section 6. Finally, the conclusion is in Section 7.

\section{Related Works}

In this section we discuss some routing protocols which have been proposed in literature for both fixed and mobile sensor nodes. 


\subsection{Routing Protocols in WSNs with Fixed Nodes}

For routing in WSNs where sensor nodes are fixed in their locations, many efforts have been focused on how to propose routing protocols that meet WSNs requirements to extend battery lifetime. For example, authors in [21] proposed energy efficient routing protocol based on random projection technique. Nodes are located using polar coordinates and routs for packets delivery are determined before sending packets which are compressed to eliminate the duplication of packets delivery with same contents. Authors in [22], proposed WSNs routing protocol which routs packets between $\mathrm{CHs}$ after forming the sensing field into clusters with different sizes. The shortest path and the residual energy are the main factors which are proposed to achieve fast and low energy consumption when sending packets to the BS. Authors in [23] proposed routing algorithm which is based on multiple factor for energy efficient WSNs. These factors include residual energy, end to end delay, the link quality and the shortest distance to the BS. The proposed algorithm shows high packets delivery ratio on a large scale sensing area. Greedy routing in WSNs is proposed in [24]. Authors analyzed the ratio of successful packets delivery to the BS when the sensing filed has some obstacles such as a lake. Also, authors studied the affect of packets congestion and collision on real world deliverability of data in their experiment. In order to solve the problem of packets collision and congestion in WSNs, authors in [25] proposed a game theoretical framework which focused on selecting the next hop in the routing path. In [26], a multipath opportunistic routing protocol is proposed for the purpose of solving the problem of multipath transmission where a node may die due to multiple transceivers processing when forming paths to a destination. The reliability of a single node needs to be increased in order to increase the reliability of the routing path, so the networks may ensure the scalability with less power consumption of sensor nodes. Decreasing end to end delay based on a routing protocol with minimum number of nodes when forming a path from a source node to a destination is proposed in [27]. When a node generates its packet, it will be forwarded to the next node which may have some packets in a queue which need to be forwarded. The proposed protocol ensures that when packets arrive to a node, it will find another path to the BS when the waiting time in the queue is too long. Combining between the requirements of MAC and routing layers when forming a path between a source node and a destination in WSNs is a protocol which is explained in [28]. Authors proposed two MAC algorithms in order to evaluate the channel quality. For the routing layer, authors proposed a routing algorithm which aims to form multiple paths between two nodes taking into consideration the shortest path and the link quality which is measured by the proposed MAC algorithms. How to construct a cluster and form a routing tree of sensor nodes in WSNs is the technique which is proposed in [29]. The proposed technique evaluates different scenarios such as distributing sensor nodes randomly or uniformly with multiple numbers of sensor nodes (i.e. 200, 400 and etc.). 


\subsection{Routing Protocols in WSNs with Fixed Nodes}

In this subsection we discuss the routing techniques based on mobile topology of nodes which are distributed in a sensing filed. In literature, many researchers have been focused on mobile nodes and their abilities to extend the lifetime of sensor nodes.

Authors in [30] redefined the lifetime of WSNs as the time for the depleted energy of mobile source nodes. Authors assumed that mobile nodes are working as relay nodes which generate packets and transfer them to the next node or to the fixed BS. All nodes send their packets only in their transmission range which reduce the power needed to send, receive and sense packets. In [31], routing in low power and lossy network such as WSNs based on mobile nodes in industrial applications is discussed extensively. The authors proposed a position determination technique for certain mobile nodes which are working beside fixed nodes in order to increase the reliability of transferring packets to the BS. Since the proposed position based technique for the mobile nodes focus on location of each sensor, authors compare their performance results with well-known geographical routing techniques. Forming clusters for mobile nodes in WSNs and proposing cluster based routing technique is discussed in [32]. The technique is based on adaptive TDMA (i.e. Time Division Multiple Access). Each mobile sensor node sends their packets during its assigned timeslot. Not only the fixed node inside a cluster has the ability to send its packet but all mobile nodes which enter the cluster can send its packet as long as there is available timeslot in TDMA scheduling mechanism. The proposed technique performance is compared with well-know mobile LEACH protocol (i.e. Low Energy Adaptive Clustering Hierarchy), and the packets loss has been reduced by around 25\%. Creation of active area between a source and a destination for routing packets of mobile nodes is discussed in [33]. Mobile nodes are tuned between sleep and active modes in order to consume low energy. Many factors have been studied to determine the direction of mobile nodes, their speed and residual energy in order to provide maximum connectivity.

\section{Network Transmission Model}

In this section, we explain the network transmission model for our proposed EERSM technique. We design a WSN which consists of many sensor nodes which are distributed randomly in a sensing filed. Sensor nodes consist of stationary which are fixed in their locations and mobile nodes which are located in the intersection areas between clusters. Figure 2 illustrates the network model. The network transmission mode includes the following assumptions:

- The sensing field is divided into intersected clusters.

- The nodes are distributed randomly in the sensing filed which includes stationary nodes and mobile nodes located in the intersected areas.

- Every cluster has two or three intersected areas. Every intersected area has one gateway node and pre-assigned mobile node as shown in Figure 2. 


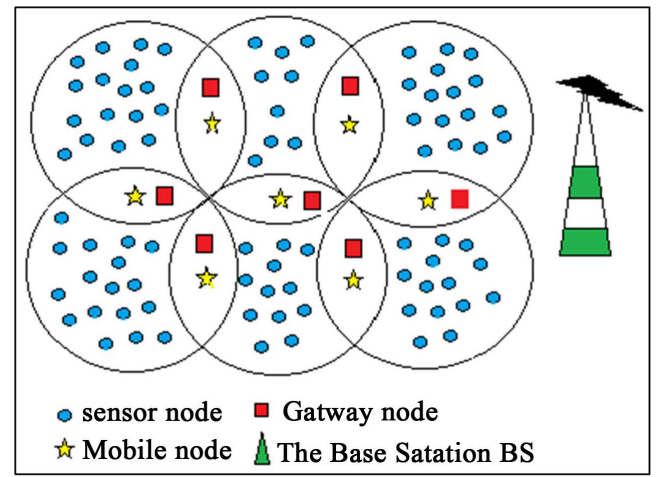

Figure 2. Network transmission model of proposed EERSM.

- The transmission like between any two sensor nodes is bidirectional.

- Every node has the ability to determine the distance to the next hop forwarding and hence control the transmission power.

- All sensor nodes have the same initial energy and the BS has ultimate power.

- All sensor node consume power for transmitting, receiving and relaying packets [34] (i.e. Total energy consumed for each sensor node $\left(E_{\text {Total }}=E_{T}+E_{R}+E_{\text {relay }}\right)$, where $E_{T}$ is energy consumed due to transmitting 1 bits of a packet, which is equal to the total of electrical circuit energy consumption $\left(E_{\text {cir }}\right)$ plus amplifier energy consumption $\left(E_{\text {amp }}\right) . E_{R}$ is energy consumed due to receiving 1 bits of a packet, which is equal to the total of electrical circuit energy consumption $\left(E_{c i r}\right)$ plus data aggregation energy consumption $\left(E_{A G}\right)$.

- All sensor nodes stay in sleeping mode when they don't have packets to send, and turn on the transceiver (active mode) only when they have packets to receiver or send. Hence leading to further power saving.

The First order radio model is assumed for packets transmission [35]. This radio model is based on the distance ( $d$ ) between a source node (e.g. node $\mathrm{X}$ ) and a destination (e.g. node $\mathrm{Y}$ ). If $d$ between $\mathrm{X}$ and $\mathrm{Y}$ is less than a threshold level ( $\left.d_{t h r}\right)$, then the free space propagation model is used). If $d$ between $\mathrm{X}$ and $\mathrm{Y}$ is greater than a threshold level $\left(d_{t h r}\right)$, then the multipath propagation model is used.

\section{Proposed EERSM Algorithm}

In the proposed EERSM algorithm sensor nodes are distributed randomly in sensing area (i.e. a harsh environment). This sensing area (i.e. $G=\{x, y\}$; where $x$ and $y$ represent the dimensions of the sensing area) include sensor nodes which perform monitoring or detecting physical phenomena. Theses sensor nodes are divided into a large number of stationary nodes and less number of mobile nodes. For better energy efficiency both kinds of sensor nodes are deigned to be in sleeping phase all the time unless they have packets to send, receiver or relay which shift the transceiver of nodes to the active mode. Mobile nodes move in two directions at angle $\varphi=0^{\circ}$ (or straight ahead) and $90^{\circ}$ at velocity $V_{m}$ (more 
details are explained in the following subsections). The BS is located in stationary location (i.e. $\left.B S=\left(x_{B S}, y_{B S}\right)\right)$. In addition, gateway nodes $\left(g_{k}\right)$; where $k$ is the gateway node number), for data aggregations are located in $g w_{k}=\left(x_{G W_{k}}, y_{G W_{k}}\right)$.

The proposed EERSM has two phases (i.e. initialization and data transmission) which are discussed in the following subsections.

\subsection{Initialization Phase}

At the first stage of the proposed EERSM technique, the sensing field which includes stationary and mobile nodes is divided into equal size clusters (i.e. equal size circles). And the distance between the centres of two neighbouring clusters $\left(l_{c i r}\right)$ is the same for all clusters. Hence, the intersected areas between circles or clusters have similar sizes which can be calculated as explained in [36]. If the distance between the centres of two neighbouring circles $l_{c i r}=0$, then the area of intersected sector is $\pi r^{2}$ (i.e. the two neighbouring clusters are exactly overlapped); where $r$ is the radii of a cluster. If the distance between the centres of two neighbouring circles $l_{\text {cir }}$ is greater than the double of the radius (or $2 r$ ) for one of the similar size circles, then the two neighbouring circles don't overlapped and the area of the intersected sector is 0. As shown in Figure 3, the mobile nodes are located in the intersected areas (sectors) between clusters. First we need to figure out the area of sectors (let's denote it $\Lambda$ ), and then we need to calculate the area of the tiny part of that sectors (let's denote it $\partial$ ). This can be done by subtracting the area of corresponding triangle (let's denote it $\hat{E}$ ) as follows:

$$
\partial=\Lambda-\widehat{E}
$$

In our works it is very important to determine the area of sectors in order to check the current locations of the mobile nodes which are moving as explained at angles $\varphi=0^{\circ}$ or $90^{\circ}$. The area of a sector $(\Lambda)$ is equal to $(\varphi / 2) r^{2}$; where $\varphi$ is the angle of the sector [36]. In Figure 3, it is shown that the height of the triangle is equal to $l_{\text {cir }} / 2$. Now, let's divide the triangle into two equal sizes triangles as shown in Figure 4. Then $\varphi$ becomes $\varphi / 2$. Using trigonometry as follows:

$$
\cos (\varphi / 2)=\left(l_{\text {cir }} / 2\right) / r=l_{\text {cir }} / 2 r
$$

In order to figure out $\varphi$, Equation (1) can be rewritten as follows:

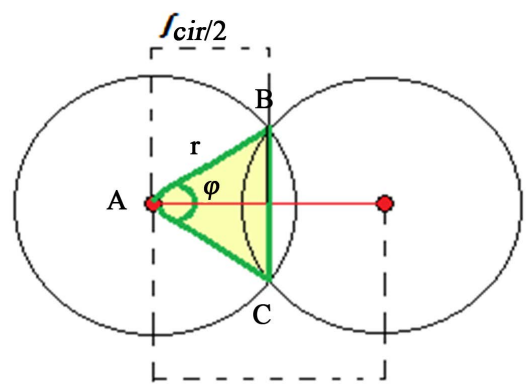

fcir

Figure 3. Calculating sectors areas of clusters. 

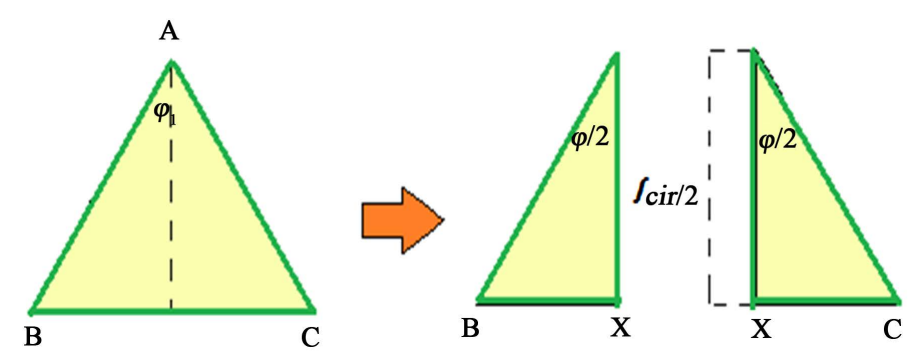

Figure 4. Calculating areas of triangles $\partial$.

$$
\varphi=2 \cos ^{-1}\left(l_{\text {cir }} / 2 r\right)
$$

Now, we can calculate the area of a sector $(\Lambda)$ which is equal to $(\varphi / 2) r^{2}$ as follows:

$$
\Lambda=(\varphi / 2) r^{2}=\left(2 \cos ^{-1}\left(l_{\text {cir }} / 2 r\right) / 2\right) r^{2}=r^{2} \cos ^{-1}\left(l_{\text {cir }} / 2 r\right)
$$

Now, we need to find out the area of the tiny part of the sectors. The area of $\widehat{E}$ in Figure 4 is equal to $(1 / 2)\left(r_{B C}\right)\left(l_{\text {cir }} / 2\right)$; where $r_{B C}$ is the length of the line between angle $\mathrm{B}$ and $\mathrm{C}$ in Figure 4. Using Pythagorean theorem [36] to calculate $r_{B C}$, we can find the base of the half of the triangle (let's denote it $r_{B C} / 2$ ) as follows:

$$
\left(r_{B C} / 2\right)^{2}+\left(l_{\text {cir }} / 2\right)^{2}=r^{2}
$$

Now, let's rewrite (5) to find out $l_{\text {Base }}$ as follows:

$$
\begin{gathered}
\left(r_{B C}\right)^{2}+\left(l_{c i r}\right)^{2}=4 r^{2} \\
r_{B C}=\sqrt{4 r^{2}-l_{c i r}^{2}}
\end{gathered}
$$

The area of the tiny part of the sector $\hat{E}$ is equal to $(1 / 2)\left(r_{B C}\right)\left(l_{\text {cir }} / 2\right)$ or:

$$
\widehat{E}=(1 / 2)\left(\sqrt{4 r^{2}-l_{\text {cir }}^{2}}\right)\left(l_{\text {cir }} / 2\right)
$$

Finally, let's call Equation (1) to calculate the intersected area between clusters as follows:

$$
\begin{gathered}
\partial=\Lambda-\widehat{E} \\
\partial=\left[r^{2} \cos ^{-1}\left(l_{c i r} / 2 r\right)\right]-\left[(1 / 2)\left(\sqrt{4 r^{2}-l_{c i r}^{2}}\right)\left(l_{c i r} / 2\right)\right]
\end{gathered}
$$

In our proposed EERSM, the BS starts sending broadcast messages to all sensor nodes which are distributed randomly in the sensing filed. These broadcast messages reach to all mobile and stationary nodes which respond by sending their locations, unique ID numbers, and the initial energy levels. The BS uses the information to determine the distance between any node and the BS or the distance between any node and the fixed gateway nodes inside intersected areas. Also, the ID numbers are used to identify the mobile or stationary nodes in order to rout packets through clusters and their related intersected areas (i.e. we call them sectors).

In our network scenario the sensing area is divided into six clusters (i.e. $\sum_{c=1}^{6} C_{c}$ ) with seven intersected areas (sectors) (i.e. $\sum_{s=1}^{7} S_{s}$ ) which contain 
seven mobile nodes (i.e. $\left.\quad \sum_{j=1}^{7} m_{j}\right)$. This can be expressed as $\forall m_{j}\left(S_{s}\left(m_{j}\right)\right)$.

\subsection{Data Transmission Phase}

In EERSM, once the BS receives all messages from all nodes in the sensing field which specify the initial energy level, the locations of nodes and the related ID numbers, it starts receiving data packets from sensor nodes. All nodes which are located in the same cluster will sense the same physical phenomena.

At the first round, the well known Dijkstra algorithm with multi-hop transmission scenario estimates the rout to the closet gateway node which is fixed in its location and hence to the BS. The BS determines the rout and sends it to all related nodes. The related nodes construct their routing tables and update them for every packet transmission. Now, data transmission will start and nodes send their packets to the closest gateway node. In order to save energy, the gateway node will collect (aggregate) similar packets and pick only one of them while other packets will be dropped. In EERSM, the gateway node sends its packet to its related mobile node which is located in its intersected area. If the mobile node is located in the related intersected area, then the gateway node will send the packet to that mobile node to carry the packets to the next gateway node which is closest to the BS. The mobile node moves in two directions which are the straight line $\left(\varphi=0^{\circ}\right)$ or with angle $\varphi=90^{\circ}$ in order to reach to the next intersected area. The gateway node sends its related packet to the next fixed sensor node in neighbouring cluster if the mobile node is not located in the intersected area after waiting a pre-defined time threshold level (i.e. $t_{t h r}$ ). The fixed sensor node relays the packet to the closest gateway node to the BS. When the packet reaches to the closest gateway node to the BS, it will send that packet to the BS and another round will start. All data packets contain the energy levels which are extracted by the BS which notifies the sensor nodes in order to update their routing tables. The data transmission phase will be repeated periodically for all rounds.

The flowchart of EERSM is demonstrated in Figure 5.

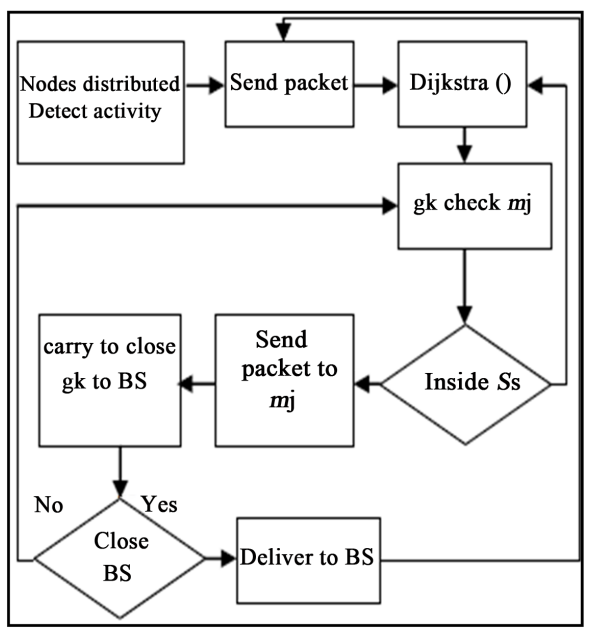

Figure 5. Flowchart of EERSM algorithm. 


\section{Proposed EERSM Algorithm Examples}

In this section we discuss various examples for the proposed EERSM algorithm for further clarification.

Example one is illustrated in Figure 6. In this figure, two sensor nodes in cluster 1(i.e. $C_{1}$ ) sense the same physical phenomena. They turn on their transceiver in order to send their packets to the closest gateway node $g_{1}$. They always follow multi-hop transmission scenario and Dijkstra algorithm to find the closest gateway node (i.e. $g_{1}$ ). The gateway node $g_{1}$ collects these packets and selects only one of them for further transmission and drops the other packet. The gateway node $g_{1}$ uses the positioning system (i.e. GPS) to determine the location of the mobile node $m_{1}$ which is assigned for the corresponding intersected area (i.e. $S_{1}$ ). The gateway node found that the mobile node $m_{1}$ is located in the intersected area $S_{1}$. So the packet will be transmitted to the mobile node which is responsible for carrying that packet to the closest (next) intersected area (i.e. $S_{2}$ ) to the BS. The movement of the mobile node follows the straight line direction for a distance equal to the diameter of one cluster (circle). When the mobile node arrives to the next intersected area (i.e. $S_{2}$ ), it will send the packet to the corresponding gateway node $g_{2}$ for that sector (i.e. $S_{2}$ ). Now, the gateway node $g_{2}$ also finds that the mobile node $m_{2}$ is located in $S_{2}$, so the packet will be transmitted to $m_{2}$ which is responsible for carrying the packet to $S_{3}$ since it is the closest intersected area to the BS. Here, the movement of the mobile node follows angle $\varphi=90^{\circ}$. Now, $m_{2}$ will send the packet to $g_{3}$ which delivers the packet to the BS.

Example two is illustrated in Figure 7. One sensor node in cluster 6 (i.e. $C_{6}$ ) sends its packet after detecting physical phenomena which needs to be transmitted. Multi-hop transmission scenario with Dijkstra algorithm is used in order to carry the packet to the closest gateway node to the BS (i.e. $g_{6}$ ). The packet is arrived to $g_{6}$ which tries to send the packet to $m_{6}$. However, $g_{6}$ waits a pre-defined time threshold level $t_{t h r}$ since the mobile node is not located in $S_{6}$. Now, the $g_{6}$ will send the packet using multi-hop transmission with Dijkstra algorithm to the closest gateway node to the BS (i.e. $g_{7}$ ). The packet will be relayed through the

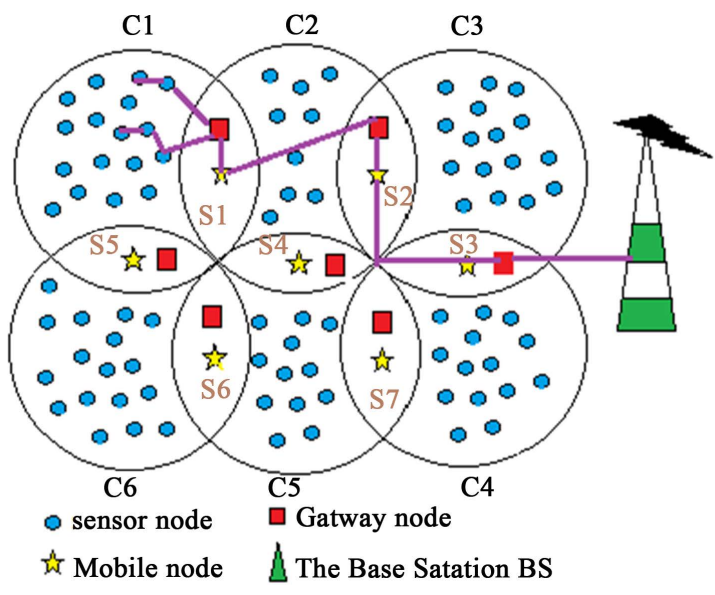

Figure 6. Example One for EERSM algorithm. 


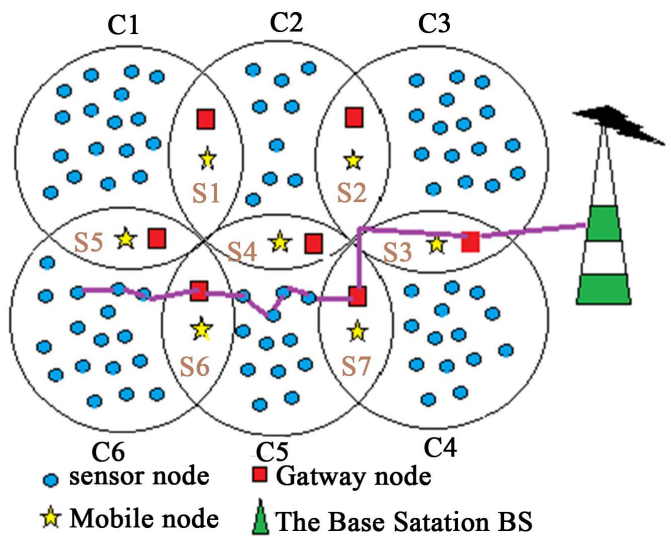

Figure 7. Example two for EERSM algorithm.

sensor node in cluster $5\left(C_{5}\right) . g_{7}$ receives the transmitted packet and check the GPS to find out the location of $m_{7}$ which is located inside the intersected area $S_{7}$. The packet will be transmitted to $m_{7}$ which moves at angle $\varphi=90^{\circ}$ in order to carry the packet to $g_{3}$. Since $g_{3}$ is the closest gateway node to the BS, it will send the packet to its final destination.

The proposed EERSM algorithm is given below. We explain some notations, the initial phase and the data transmission phase.

Energy Efficient Routing with Stationary and Mobile nodes (EERSM) Algorithm

\section{Notations:}

- $\quad N_{p} m_{p} g_{k} C_{\mathcal{c}} S_{s}$ stationary nodes, mobile nodes, gateway nodes, clusters and intersected areas, respectively.

- $i, j, k, c, s$. the number of stationary nodes, mobile nodes, gateway nodes, clusters and intersected areas, respectively.

- $G=\{x, y\}$ : the sensing area

- $\quad x, y$ : the dimensions of the sensing area.

- $B S=\left(x_{B S}, y_{B S}\right)$ : the stationary location of the base station.

- $g w_{k}=\left(x_{G W_{k}}, y_{G W_{k}}\right):$ the stationary location of the gateway node.

- $\quad m_{x, y, j}=\left(x_{m_{j}}, y_{m_{j}}\right):$ the current location of the mobile node.

- $\quad l_{c i r}$ : the distance between the centres of two clusters.

- $t_{t h r}:$ a pre-defined time threshold level.

- $r$. the radii of a cluster.

- $\mathfrak{E}$ : nodes detect activity.

- $\tau$ : the waiting time to detect mobile node arrival.

\section{Initialization Phase:}

1) Deploy all stationary $N_{i}$, mobile $m_{j}$ and gateway $g_{k}$ nodes in the sensing field $G=\{x, y\}$.

2) The BS send broadcast messages to all nodes in $G=\{x, y\}$.

3) All $N_{i}, m_{j}$ and $g_{k}$ respond by sending the locations (i.e. $N_{x, y}=\left(x_{N_{i}}, y_{N_{i}}\right)$, $m_{x, y, j}=\left(x_{m_{j}}, y_{m_{j}}\right)$ and $g w_{k}=\left(x_{G W_{k}}, y_{G W_{k}}\right)$, the Initial energy $E_{i n t}$ and the node number (i.e. $i, j, k$, respectively) 
4) Forming clusters $C_{c}$ with equal intersected areas $S_{s}$ contain one $g_{k}$ for each $S_{s}$.

5) All gateway nodes $g_{k}$ send their locations to $N_{i}$ in its cluster.

6) Calculate the areas of the sectors $\partial$ (intersected areas) between clusters to be equal

$$
\partial=r^{2} \cos ^{-1}\left(l_{c i r} / 2 r\right)-(1 / 2)\left(\sqrt{4 r^{2}-l_{c i r}^{2}}\right)\left(l_{c i r} / 2\right)
$$

7) Locate one mobile node $m_{x, y, j}=\left(x_{m_{j}}, y_{m_{j}}\right)$ in each intersected area $S_{s}$ which has area $=\partial$.

\section{Data Transmission Phase:}

for round $=0$ to 2000 do

$N i_{\text {mode }}=0$; all stationary nodes in sleeping mode.

If $N_{i}=\mathfrak{E}$; detect activity

$N i_{\text {mode }}=1$; turn on the transceiver for transmission.

Call Dijkstra algorithm ( ) to nearest $g w_{k}=\left(x_{G W_{k}}, y_{G W_{k}}\right)$

If $(k=1 \| k=6)$

If $\left(m_{x, y, 1}=\left(x_{m_{1}}, y_{m_{1}}\right) \in S_{1} \| m_{x, y, 6}=\left(x_{m_{6}}, y_{m_{6}}\right) \in S_{6}\right.$; mobile node located in intersected area $S_{1}$ or $S_{6}$

$m_{1}$ or $m_{6}$ move to $S_{2}$ or $S_{7}$; mobile node move to

intersected areas $S_{2}$ or $S_{7}$

packet $=1$; send packet to $g_{2}$ or $g_{7}$

If $\left(m_{x, y, 2}=\left(x_{m_{2}}, y_{m_{2}}\right) \in S_{2} \| m_{x, y, 7}=\left(x_{m_{7}}, y_{m_{7}}\right) \in S_{7}\right)$

$m_{2}$ or $m_{7}$ move to $S_{3}$; mobile node move to

intersected areas $S_{3}$.

packet $=1$; send packet to BS.

Else

Wait $t_{t h r}$;

If $\left(\tau>t_{t h r}\right)$

Dijkstra ( ) to nearest $g_{2}$ or $g_{7}$

packet $=1$; send packet to $g_{2}$ or $g_{7}$

If $\left(m_{x, y, 2}=\left(x_{m_{2}}, y_{m_{2}}\right) \in S_{2} \quad \| m_{x, y, 7}=\left(x_{m_{7}}, y_{m_{7}}\right) \in S_{7}\right)$

$m_{2}$ or $m_{7}$ move to $S_{3}$; mobile node move to

intersected areas $S_{3}$

packet $=1$; send packet to BS.

Else

Dijkstra ( ) to nearest $g_{3}$

packet $=1$; send packet to BS

End If

Else

$m_{x, y, 2}=\left(x_{m_{2}}, y_{m_{2}}\right) \in S_{2} \quad \| m_{x, y, 7}=\left(x_{m_{7}}, y_{m_{7}}\right) \in S_{7} ; m_{2}$ or $m_{7}$ arrive to $S_{2}$ or $S_{7}$ $m_{2}$ or $m_{7}$ move to $S_{3}$; mobile node move to

intersected areas $S_{3}$.

packet $=1$; send packet to $g_{3}$ 
packet $=1$; send packet to BS.

End If

End If

End If

If $(k=2 \| k=7)$

If $\left(m_{x, y, 2}=\left(x_{m_{2}}, y_{m_{2}}\right) \in S_{2} \| m_{x, y, 7}=\left(x_{m_{7}}, y_{m_{7}}\right) \in S_{7}\right)$; mobile node located in the intersected area $S_{2}$ or $S_{7}$

$m_{2}$ or $m_{7}$ move to $S_{3}$; mobile node move to intersected $S_{3}$.

packet $=1$; send packet to $g_{3}$

packet $=1$; send packet to $\mathrm{BS}$

Else

Wait $t_{t h r}$;

If $\left(\tau>t_{t h r}\right)$

Dijkstra ( ) to nearest $g_{3}$

packet $=1$; send packet to $g_{3}$

packet $=1$; send packet to BS

End If

End If

If $(k=4)$

If $\left(m_{x, y, 4}=\left(x_{m_{4}}, y_{m_{4}}\right) \in S_{4}\right)$; mobile node located in the intersected area $S_{4}$

$m_{4}$ move to $S_{3}$; mobile node move to intersected areas $S_{3}$.

packet $=1$; send packet to $g_{3}$

packet $=1$; send packet to BS

Else

Wait $t_{t h r}$;

If $\left(\tau>t_{t h r}\right)$

Dijkstra ( ) to nearest $g_{3}$

packet $=1$; send packet to $g_{3}$

packet $=1$; send packet to BS

End If

End If

End If

If $(k=5)$

packet $=1$; send packet to $g_{4}$

If $\left(m_{x, y, 4}=\left(x_{m_{4}}, y_{m_{4}}\right) \in S_{4}\right)$; mobile node located in the intersected area $S_{4}$

$m_{4}$ move to $S_{3}$; mobile node move to intersected areas $S_{3}$.

packet $=1$; send packet to $g_{3}$

packet $=1$; send packet to BS

Else

Wait $t_{t h r}$;

If $\left(\tau>t_{t h r}\right)$

Dijkstra ( ) to nearest $g_{3}$

packet $=1$; send packet to $g_{3}$

packet $=1$; send packet to $B S$ 
End If

End If

End If

End If

End for

\section{Performance Evaluation}

In order to analyse the performance of the proposed EERSM algorithm, we simulate its behaviour using MATLAB. Also, we compare the proposed EERSM technique with two power efficient mobile routing protocols which are explained in [31] and [32] (i.e. routing with blacklisting technique and CBR Mobile-WSN technique respectively). Both techniques use mobile nodes to achieve power efficient routing in WSNs.

The simulation setting and environment consist of 85 stationary sensor nodes, 7 gateway nodes located in the intersected areas between clusters, 7 mobile nodes, 6 clusters and the fixed BS. The sensing field $G=\{x, y\}$; where $x$ and $y$ represent the dimensions which are equal to 80 and 55, respectively. Sensor nodes are distributed randomly where every cluster has different number of stationary nodes. The $\mathrm{BS}$ is located in stationary place, i.e. $B S=\left(x_{B S}, y_{B S}\right)$; where $x_{B S}$ and $y_{B S}$ represent the dimensions which are equal to 70 and 27.5. The gateway nodes are located in the intersected areas between clusters which have similar diameters, i.e. $20 \mathrm{~m}$. The locations of gateway nodes $g w_{i}=\left(x_{G W_{i}}, y_{G w_{i}}\right)$ are given which are equal to $g w_{1}=(25,45), g w_{2}=(45,45), g w_{3}=(60,25)$, $g w_{4}=(35,25), g w_{5}=(20,25), g w_{6}=(25,20), g w_{7}=(45,20)$. We run the simulation for 2000 rounds, and every time a sensor node generate a packet it will be delivered to the closest gatway node as explained earlier using Dijkstra algorithm with multi-hop transmission. The packet size is 500 bits.

In order to calculate the consumed energy we assume the following:

- The initial energy for any sensor node is equal to $1 \mathrm{~J}$ (i.e. $E_{\text {int }}$ )

- The transmitter energy for electrical circuit $\left(E X_{c i r}\right)=30 \mathrm{~nJ} / \mathrm{bit}$,

- The energy consumed due to data aggregation $\left(E X_{A G}\right)=10 \mathrm{~nJ} / \mathrm{bit}$,

- The transmitter energy for amplifier $\left(E X_{\text {amp }}\right)=5 \mathrm{pJ} / \mathrm{bit} / \mathrm{d}^{2}$ and 0.020 $\mathrm{pJ} / \mathrm{bit} / \mathrm{d}^{4}$

- The receiver energy for electrical circuit $\left(E Y_{\text {cir }}\right)=30 \mathrm{~nJ} / \mathrm{bit}$.

The first performance metric we discuss is the number of hops counted when sending packets from a source node to the destination (i.e. BS). We run the simulation based on our assumptions and the simulation parameters on all network scenarios of the proposed EERSM technique, routing with blacklisting technique and CBR Mobile-WSN technique. We run the simulation for the same number of sensor nodes in the three network scenarios for the three techniques. We found that the proposed EERSM technique needs less number of bops when sending packets to the BS. This is due to the capability of mobile nodes to rout packets from one intersected area to another without the need for relaying pack- 
ets thought many sensor nodes in multi-hop forwarding. It is followed by CBR Mobile-WSN technique which is closed to the number of hops utilized in EERSM technique, while routing with blacklisting technique uses many sensor nodes in multi-hop forwarding when sending their packets to the BS. Figure 8 shows this performance metric the number of hops needed for the three techniques over different number of nodes in the simulation.

The second performance metric is the average residual energy. The forwarding phase in routing with blacklisting technique needs every node to search for their nearest node in order to turn on the transceiver and send the packets to a centralized sink node. In addition; mobile nodes move randomly around the sink node in order to carry packets. However, this scenario needs many control messages to be sent between the sink node and the mobile nodes in order to detect the right mobile node to carry the packets. This makes the energy consumption increase. Also, sensor nodes in CBR Mobile-WSN technique need to search for a free cluster head in order to avoid packets loss which increases the energy consumption. In CBR Mobile-WSN technique many control messages need to be sent in order to give a permission for a mobile node to enter a cluster which consume more power, while in the proposed EERSM technique the mobile nodes move from one intersected area to another without the necessity to send control messages. Mobile nodes deliver their packets to the right position (i.e. the right intersected area) and then return to their pre-assigned positions in order to carry more packets. Figure 9 shows the residual energy for the three techniques verses the simulation rounds.

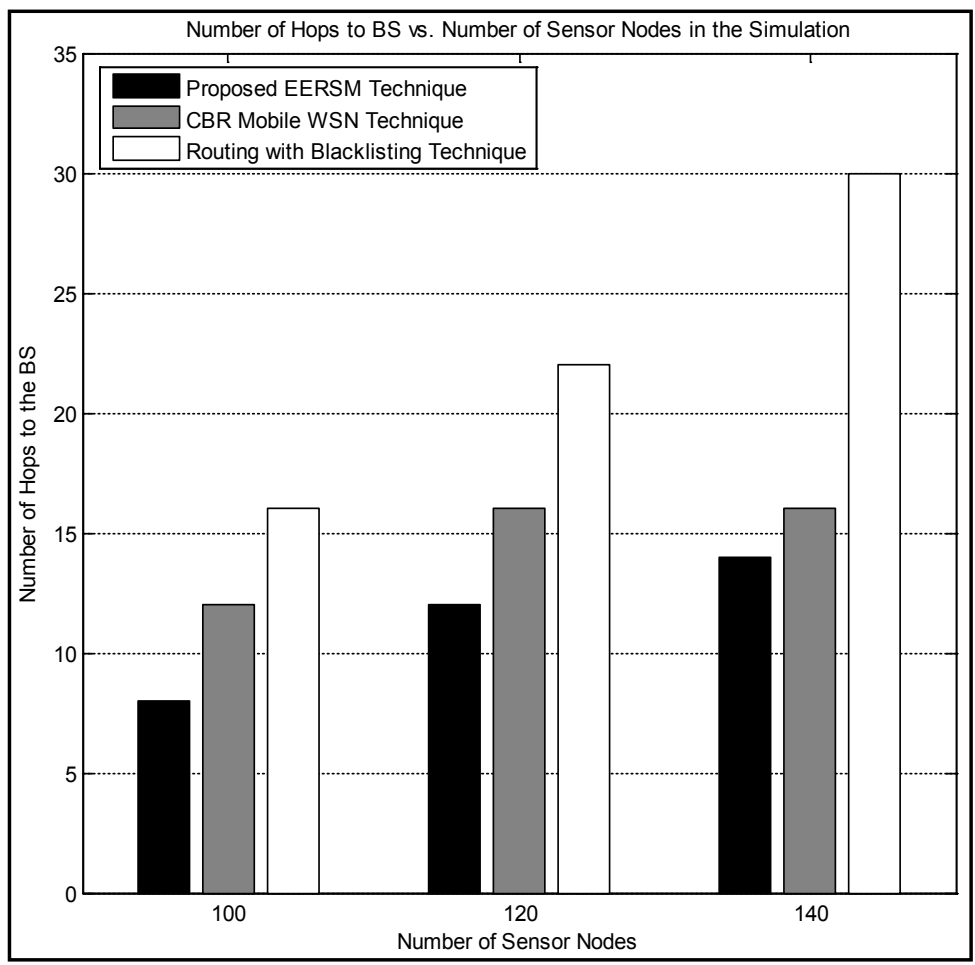

Figure 8. Number of hops counted when sending packets to the BS. 
The third performance metric is the throughput or the number of packets which are arrived successfully to the BS. Due to the fact that our proposed EERSM has longer lifetime as we will discuss in the following performance metric, nodes will still send to the BS because they are still alive for longer period of time.

Thus, the proposed EERSM has a higher curve in Figure 10, followed by CBR Mobile-WSN technique.

Finally, the fourth performance metric is the network lifetime or the time interval from the first node sending their packets until the last node becomes dead. In Figure 11, the stability period of proposed EERSM exceeds routing with blacklisting technique and CBR Mobile-WSN technique by 400 and 200 rounds respectively. The first node become dead in blacklisting technique routing at round 410, while The first node become dead in CBR Mobile-WSN technique at round 588. This is expected results due to the fact that less number of hops involved when sending packets to The BS. Most stationary sensor nodes in the proposed EERSM technique are staying in a sleep mode, and only turn on the transceivers when having packets need to be relayed through them. The majority of transmission depends on the mobile nodes which are moving in their pre-determined directions.

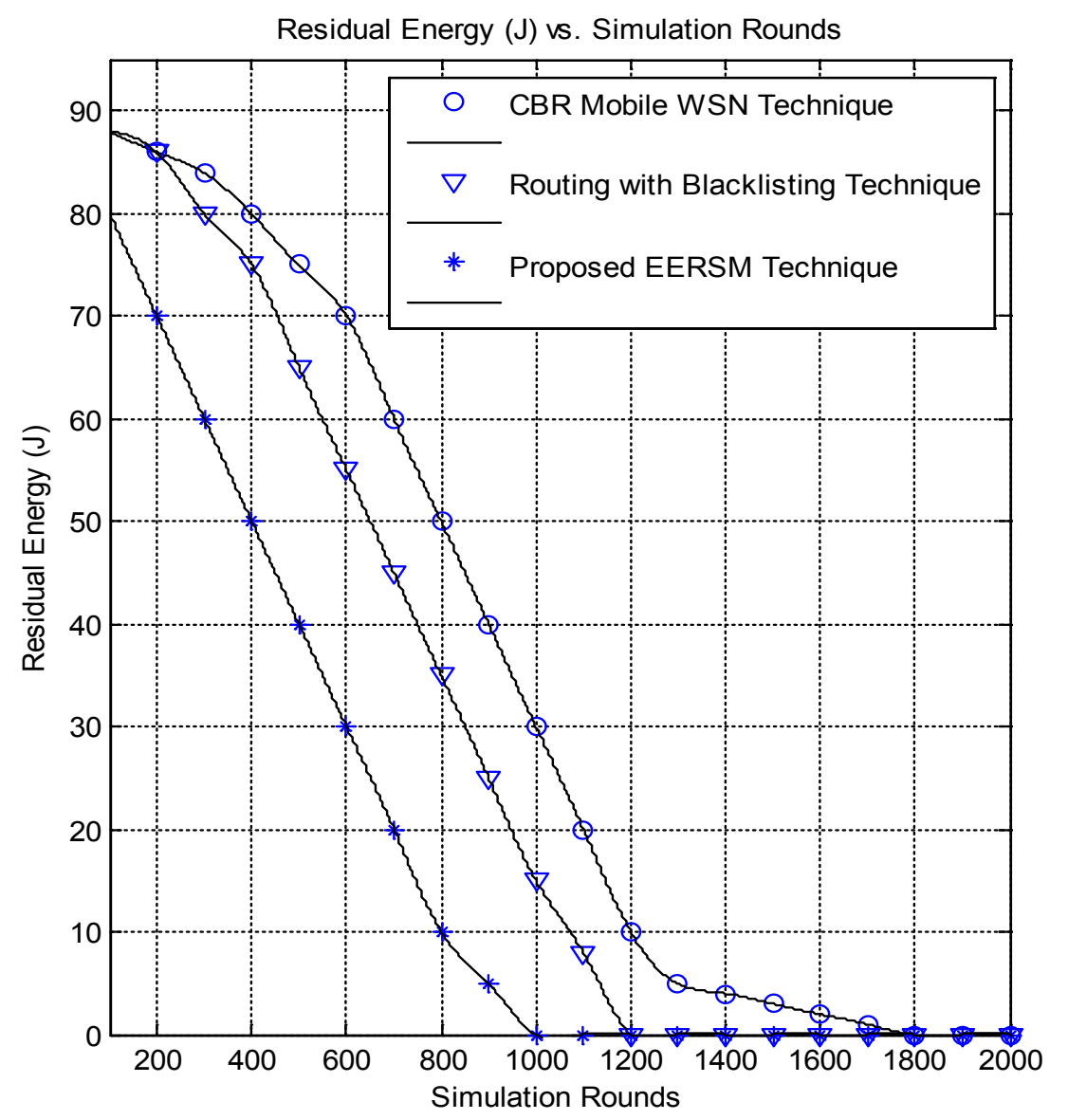

Figure 9. The residual energy verses the simulation rounds. 


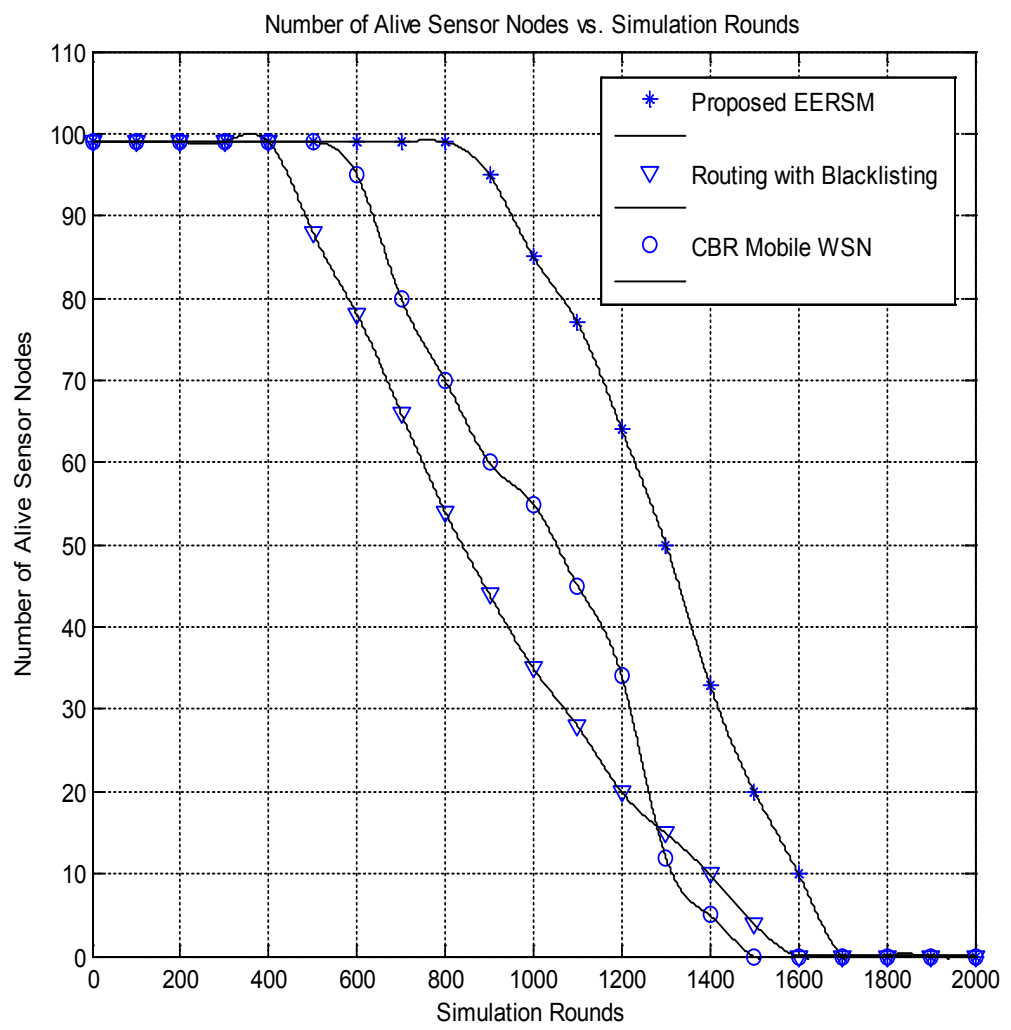

Figure 10. The throughput verses the simulation rounds.

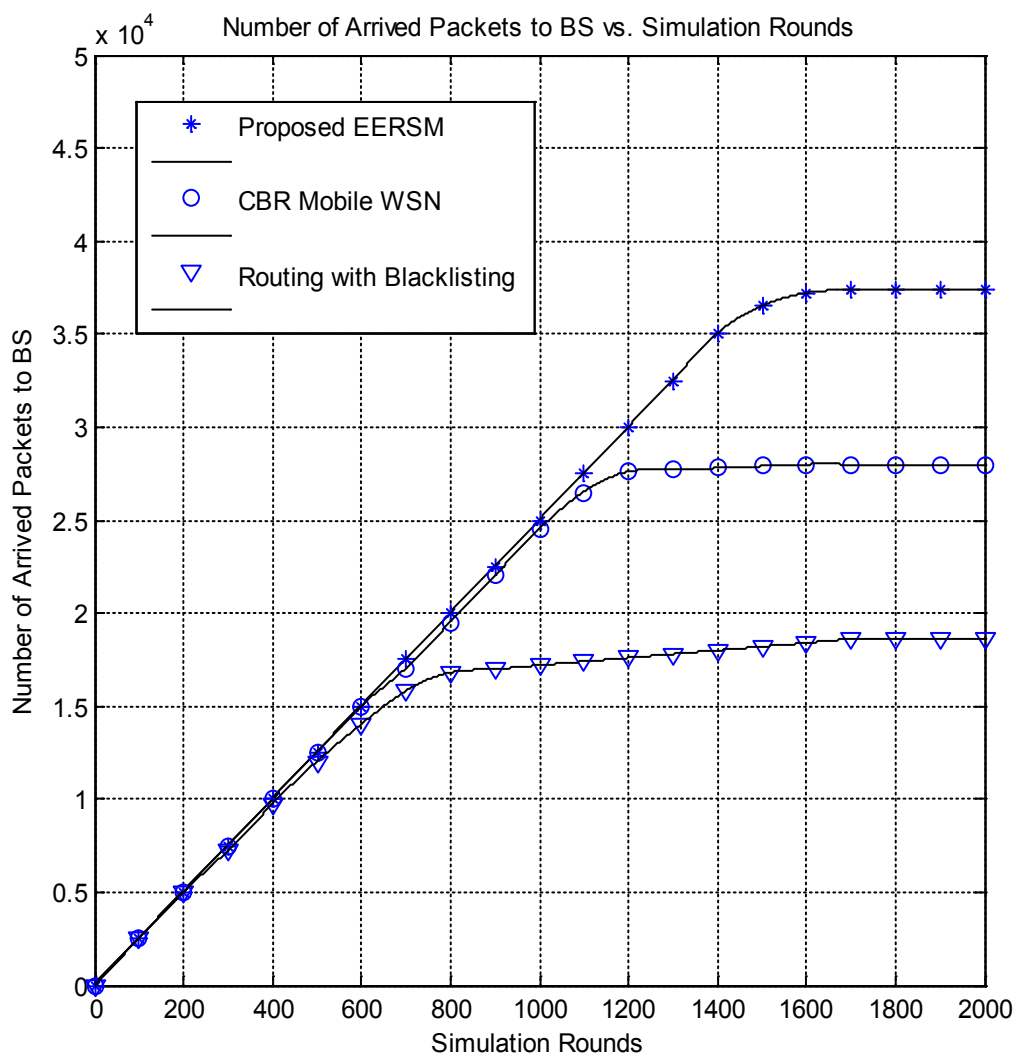

Figure 11. The lifetime of the networks verses the simulation rounds. 


\section{Conclusion}

The proposed EERSM routing technique combines the advantages of relaying packets through stationary sensor nodes and pre-assigned locations of mobile nodes. Less number of packets will be relayed using the stationary sensor nodes, and the mobile nodes don't consume too much power to for control messages. Multi-hop forwarding is used in EERSM in order to accelerate packets delivery and clusters are formed not to nominate cluster head and consume power for nomination procedures, but it is utilized in order to determine the location of mobile nodes in the intersected areas between clusters and hence to use less number of hops to packets transmission. The number of packets arrived successfully to the BS, the residual energy and the network lifetime are performance metrics which are evaluated for the proposed EERSM and compared with similar power efficient routing for mobile nodes in WSNs. From the simulation results, the proposed EERSM can be considered as energy efficient technique in multi-hop transmission scenario for stationary/mobile nodes of WSNs.

\section{Conflicts of Interest}

The author declares no conflicts of interest regarding the publication of this paper.

\section{References}

[1] Bhushan, B. and Sahoo, G. (2017) A Comprehensive Survey of Secure and Energy Efficient Routing Protocols and Data Collection Approaches in Wireless Sensor Networks. International Conference on Signal Processing and Communication, Coimbatore, 294-299. https://doi.org/10.1109/CSPC.2017.8305856

[2] Zhang, Z., Mehmood, A., Shu, L., Huo, Z., Zhang, Y. and Mukherjee, M. (2018) A Survey on Fault Diagnosis in Wireless Sensor Networks. IEEE Access, 6, 11349-11364. https://doi.org/10.1109/ACCESS.2018.2794519

[3] Sun, N., Xu, J., Wei, H., Miao, H. and Wang, J. (2017) A Network State Based Reliability Evaluation Model for WSNs. 18th IEEE/ACIS International Conference on Software Engineering, Artificial Intelligence, Networking and Parallel/ Distributed Computing, Kanazawa, 303-308. https://doi.org/10.1109/SNPD.2017.8022737

[4] Usman, M., Yang, N., Jan, M. A., He, X., Xu, M. and Lam, K.M. (2018) A Joint Framework for QoS and QoE for Video Transmission over Wireless Multimedia Sensor Networks. IEEE Transactions on Mobile Computing, 17, 746-759. https://doi.org/10.1109/TMC.2017.2739744

[5] Joshi, V., Desai, O. and Kowli, A. (2017) High Accuracy Sensor Fault Detection for Energy Management Applications. IEEE International Conference on Signal Processing, Informatics, Communication and Energy Systems, Kollam, 1-6. https://doi.org/10.1109/SPICES.2017.8091363

[6] Roy, S. and Nene, M.J. (2015) A Security Framework for Military Application on Infrastructure Based Wireless Sensor Network. IEEE International Conference on Research in Computational Intelligence and Communication Networks, Kolkata, 369-376.

[7] Lee, S.H., Lee, S., Song, H. and Lee, H.S. (2009) Wireless Sensor Network Design for Tactical Military Applications: Remote Large-Scale Environments. IEEE Military 
Communications Conference, Boston, 1-7.

https://doi.org/10.1109/MILCOM.2009.5379900

[8] Diamond, S.M. and Ceruti, M.G. (2007) Application of Wireless Sensor Network to Military Information Integration. 5th IEEE International Conference on Industrial Informatics, Vienna, 317-322. https://doi.org/10.1109/INDIN.2007.4384776

[9] Wang, Y., Rajib, S.M., Collins, C. and Grieve, B. (2018) Low-Cost Turbidity Sensor for Low-Power Wireless Monitoring of Fresh-Water Courses. IEEE Sensors Journal, 18, 4689-4696. https://doi.org/10.1109/JSEN.2018.2826778

[10] Khan, R., Ali, I., Zakarya, M., Ahmad, M., Imran, M. and Shoaib, M. (2018) Technology-Assisted Decision Support System for Efficient Water Utilization: A Real-Time Testbed for Irrigation Using Wireless Sensor Networks. IEEE Access, 6, 25686-25697. https://doi.org/10.1109/ACCESS.2018.2836185

[11] Tijero, M., et al. (2017) Vacuum Packaging and Semipassive Chips for Wireless Temperature Monitoring in Industrial Applications. IEEE Sensors, Glasgow, 1-3.

[12] Farag, H., Gidlund, M. and Osterberg, P. (2018) A Delay-Bounded MAC Protocol for Mission- and Time-Critical Applications in Industrial Wireless Sensor Networks. IEEE Sensors Journal, 18, 2607-2616. https://doi.org/10.1109/JSEN.2018.2793946

[13] Li, M., Hua, C., Chen, C. and Guan, X. (2017) Application-Driven Virtual Network Embedding for Industrial Wireless Sensor Networks. IEEE International Conference on Communications, Paris, 1-6. https://doi.org/10.1109/ICC.2017.7996431

[14] Nisar, K., Ibrahim, A.A.A., Wu, L., Adamov, A. and Deen, M.J. (2016) Smart Home for Elderly Living Using Wireless Sensor Networks and an Android Application. IEEE 10 th International Conference on Application of Information and Communication Technologies, Baku, 1-8. https://doi.org/10.1109/ICAICT.2016.7991655

[15] Irmak, E., Köse, A. and Göçmen, G. (2016) Simulation and ZigBee Based Wireless Monitoring of the Amount of Consumed Energy at Smart Homes. IEEE International Conference on Renewable Energy Research and Applications, Birmingham, 1019-1023. https://doi.org/10.1109/ICRERA.2016.7884488

[16] Freitas, D.J., Marcondes, T.B., Nakamura, L.H.V., Ueyama, J., Gomes, P.H. and Meneguette, R.I. (2015) Combining Cell Phones and WSNS for Preventing Accidents in Smart-Homes with Disabled People. 7 th International Conference on New Technologies, Mobility and Security, Paris, 1-5. https://doi.org/10.1109/NTMS.2015.7266477

[17] Tennina, S., et al. (2016) WSN4QoL: WSNs for Remote Patient Monitoring in e-Health Applications. IEEE International Conference on Communications, Kuala Lumpur, 1-6. https://doi.org/10.1109/ICC.2016.7511597

[18] Shih, Y.Y., Hsiu, P.C. and Pang, A.C. (2019) A Data Parasitizing Scheme for Effective Health Monitoring in Wireless Body Area Networks. IEEE Transactions on Mobile Computing, 18, 13-27. https://doi.org/10.1109/TMC.2018.2830779

[19] Latif, S., Afzaal, H. and Zafar, N.A. (2018) Intelligent Traffic Monitoring and Guidance System for Smart City. International Conference on Computing, Mathematics and Engineering Technologies, Sukkur, 1-6.

[20] Zhou, J., Chen, C.L.P. and Chen, L. (2013) A Small-Scale Traffic Monitoring System in Urban Wireless Sensor Networks. IEEE International Conference on Systems, Man, and Cybernetics, Manchester, 4929-4934. https://doi.org/10.1109/SMC.2013.842

[21] Qiao, J. and Zhang, X. (2018) Polar Coordinate-Based Energy-Efficient-Chain Routing in Wireless Sensor Networks Using Random Projection. IEEE Access, 6, 
21275-21286. https://doi.org/10.1109/ACCESS.2018.2824245

[22] Cisse, C.S.M., Bennis, I., Ayaida, M. and Sarr, C. (2017) Gateway Selection Technique for Efficient Multi-Hop Routing in Wireless Sensor Networks. International Conference on Wireless Networks and Mobile Communications, Rabat, 1-7. https://doi.org/10.1109/WINCOM.2017.8238144

[23] Lai, X., Ji, X., Zhou, X. and Chen, L. (2018) Energy Efficient Link-Delay Aware Routing in Wireless Sensor Networks. IEEE Sensors Journal, 18, 837-848. https://doi.org/10.1109/JSEN.2017.2772321

[24] Liu, J., et al. (2018) Characterizing Data Deliverability of Greedy Routing in Wireless Sensor Networks. IEEE Transactions on Mobile Computing, 17, 543-559. https://doi.org/10.1109/TMC.2017.2737005

[25] Attiah, A., Amjad, M.F., Chatterjee, M. and Zou, C.C. (2016) An Evolutionary Game for Efficient Routing in Wireless Sensor Networks. IEEE Global Communications Conference, Washington DC, 1-7. https://doi.org/10.1109/GLOCOM.2016.7841592

[26] Kim, S., Cho, H., Yang, T., Kim, C. and Kim, S.H. (2017) Low-Cost Multipath Routing Protocol by Adapting Opportunistic Routing in Wireless Sensor Networks. IEEE Wireless Communications and Networking Conference, San Francisco, 1-6. https://doi.org/10.1109/WCNC.2017.7925730

[27] Souissi, M. and Meddeb, A. (2017) Design Optimization of Delay-Constrained Routing in Wireless Sensor Networks. IEEE 7 th Annual Computing and Communication Workshop and Conference, Las Vegas, 1-6. https://doi.org/10.1109/CCWC.2017.7868466

[28] Li, J., Xi, J. and Ren, Q. (2016) MR-MC: A Joint Routing Protocol in Wireless Sensor Networks. IEEE 18 th International Conference on High Performance Computing and Communications, Sydney, 231-236. https://doi.org/10.1109/HPCC-SmartCity-DSS.2016.0042

[29] Nie, C., Wu, H. and Zheng, W. (2016) Latency and Lifetime-Aware Clustering and Routing in Wireless Sensor Networks. IEEE 41 st Conference on Local Computer Networks, Dubai, 164-167. https://doi.org/10.1109/LCN.2016.33

[30] Pourazarm, S. and Cassandras, C.G. (2017) Optimal Routing for Lifetime Maximization of Wireless-Sensor Networks with a Mobile Source Node. IEEE Transactions on Control of Network Systems, 4, 793-804. https://doi.org/10.1109/TCNS.2016.2559018

[31] Barcelo, M., Correa, A., Vilajosana, X., Vicario, J.L. and Morell, A. (2014) Novel Routing Approach for the TSCH Mode of IEEE 802.15.14e in Wireless Sensor Networks with Mobile Nodes. IEEE 80 th Vehicular Technology Conference, Vancouver, 1-5.

[32] Awwad, S.A.B., Ng, C.K., Noordin, N.K. and Rasid, M.F.A. (2009) Cluster Based Routing protocol for Mobile Nodes in Wireless Sensor Network. International Symposium on Collaborative Technologies and Systems, Baltimore, 233-241. https://doi.org/10.1109/CTS.2009.5067486

[33] Sreejith, V., Surve, R., Vyas, N., Anupama, K.R. and Gudino, L.J. (2018) Area Based Routing Protocol for Mobile Wireless Sensor Networks. International Conference on Information Networking, Chiang Mai, 782-786.

[34] Fawzy, A.E., Shokair, M. and Saad, W. (2018) Balanced and Energy-Efficient Multi-Hop Techniques for Routing in Wireless Sensor Networks. IET Networks, 7, 33-43. https://doi.org/10.1049/iet-net.2017.0063

[35] Heinzelman, W.R., Chandrakasan, A. and Balakrishnan, H. (2000) Energy-Efficient 
Communication Protocol for Wireless Microsensor Networks. The 33rd Annual Hawaii International Conference, Hawaii, 10-11. https://doi.org/10.1109/HICSS.2000.926982

[36] Pedoe, D. (1995) Circles: A Mathematical View. The Mathematical Association of America, New York, Vol. 1, 23-109. 